\title{
Suppression of alternans and conduction blocks despite steep APD restitution: electrotonic, memory, and conduction velocity restitution effects
}

\author{
Elizabeth M. Cherry ${ }^{1}$ and Flavio H. Fenton ${ }^{1,2}$ \\ ${ }^{1}$ Hofstra University, Hempstead 11549; and ${ }^{2}$ Beth Israel Medical Center, New York, New York 10003
}

Submitted 6 August 2003; accepted in final form 22 January 2004

\begin{abstract}
Cherry, Elizabeth M., and Flavio H. Fenton. Suppression of alternans and conduction blocks despite steep APD restitution: electrotonic, memory, and conduction velocity restitution effects. Am J Physiol Heart Circ Physiol 286: H2332-H2341, 2004. First published January 29, 2004; 10.1152/ajpheart.00747.2003.-We examine the utility of the action potential (AP) duration (APD) restitution curve slope in predicting the onset of electrical alternans when electrotonic and memory effects are considered. We develop and use two ionic cell models without memory that have the same restitution curve with slope $>1$ but different AP shapes and, therefore, different electrotonic effects. We also study a third cell model that incorporates short-term memory of previous cycle lengths, so that it has a family of S1-S2 restitution curves as well as a dynamic restitution curve with slope $>1$. Our results indicate that both electrotonic and memory effects can suppress alternans, even when the APD restitution curve is steep. In the absence of memory, electrotonic currents related to the shape of the AP, as well as conduction velocity restitution, can affect how alternans develops in tissue and, in some cases, can prevent its induction entirely, even when isolated cells exhibit alternans. When short-term memory is included, alternans may not occur in isolated cells, despite a steep APD restitution curve, and may or may not occur in tissue, depending on conduction velocity restitution. We show for the first time that electrotonic and memory effects can prevent conduction blocks and stabilize reentrant waves in two and three dimensions. Thus we find that the slope of the APD restitution curve alone does not always well predict the onset of alternans and that incorporating electrotonic and memory effects may provide a more useful alternans criterion. A Data Supplement containing movies and JAVA applets is available online at http://ajpheart.physiology.org/cgi/ content/full/00747.2003/DC1.
\end{abstract}

action potential morphology; arrhythmias; reentry

FROM ITS DISCOVERY in experimental (37) and clinical (47) settings in the early 1900s and although it was considered rare until the 1940s (41), alternans has been recognized as a precursor to the induction of some ventricular arrhythmias. Some more recent clinical studies $(60,62)$ have shown a correlation between the presence of alternans in the ECG and the likelihood of developing cardiac fibrillation, suggesting that interventions that eliminate alternans may inhibit conduction blocks and, thereby, prevent fibrillation. Building on the work of Nolasco and Dahlen (51), who showed by graphical arguments how steep action potential (AP) duration (APD) restitution curves could produce alternans, much emphasis has been placed over the last decade on the slope of the APD restitution curve as one of the main factors in the induction of ventricular fibrillation (VF) both experimentally $(31,46,57)$ and numerically (Refs. 15 and 42; D. R. Chialvo, unpublished observations). This theory-derived condition for the onset of

Address for reprint requests and other correspondence: F. H. Fenton, Dept of Physics, CHPHB 102, Hofstra Univ., Hempstead, NY 11549 (E-mail: Flavio.H.Fenton@Hofstra.edu). alternans when the slope of the APD restitution curve as a function of the diastolic interval (DI) is $>1$ (here called the APD restitution condition) has prompted what has been referred to as the restitution hypothesis, which postulates $(43,52)$ that flattening the APD restitution curve inhibits alternans and subsequent conduction blocks, thereby preventing fibrillation. As an outgrowth of this hypothesis, altering the restitution curve has been proposed as a target for antifibrillatory drug design $(33,43,48)$.

However, some questions and criticisms concerning the applicability of restitution curve slopes as a predictor of fibrillation have been raised recently $(2,32,40)$, such as the existence of mechanisms for fibrillation besides alternans $(3,6$, $7,21,23,44,56,58,61)$, the existence of mechanisms for alternans other than restitution $(1,13,14,34,50,53,54,63)$, and difficulties and complexities in measuring and analyzing restitution curves $(23,40)$. Furthermore, recent studies in animal models $(2,36)$ have shown that preparations having dynamic APD restitution curves with slopes $>1$ do not necessarily exhibit alternans, as the original restitution criterion of Nolasco and Dahlen (51) would suggest, but in fact may exhibit stable behavior, even at short cycle lengths, where the restitution curve slopes are steepest. Recent theoretical studies in simulated isolated cardiac cells and one-dimensional cables have confirmed these experimental findings and have demonstrated that two important physiological characteristics, electrotonic effects $(17,18)$ and memory $(26,64)$, should be included in the alternans criterion to explain both theoretical and experimental results.

In this paper, we use a mathematical model that allows direct variation of three features not easily altered experimentally, namely, the APD and conduction velocity (CV) restitution curves and AP shape, to analyze their effects on the development of alternans. We explain under what conditions electrotonic effects and memory can suppress alternans and conduction block and show for the first time that sustained wave break may not develop in two- and three-dimensional tissue, despite APD restitution curve slope much greater than 1 .

\section{METHODS}

Because our study is focused on restitution and because the restitution hypothesis is concerned with the properties of the restitution curve, rather than the specific currents that underlie it, we selected a model (25) whose parameters, such as the opening and closing of the gate variables and the ionic conductances, can be varied to fit and reproduce specific mesoscopic characteristics, such as the APD and $\mathrm{CV}$ restitution curves, as well as the AP shape. Versions of this phenomenological ionic model have been shown previously $(8,9,23$,

The costs of publication of this article were defrayed in part by the payment of page charges. The article must therefore be hereby marked "advertisement" in accordance with 18 U.S.C. Section 1734 solely to indicate this fact. 
25) to accurately reproduce the dynamics of other numerical models such as the Beeler-Reuter model (5), the Luo-Rudy I model (49), and the model of Courtemanche et al. (16). It also has been used to reproduce experimentally obtained restitution curves for guinea pig (25) and rabbit (21) ventricular myocardium, as well as the AP shapes for canine epicardial, endocardial, and $\mathrm{M}$ cells (including appropriate spike-and-dome morphology for epicardial and $\mathrm{M}$ cells) (10).

In the model, the dynamics of the transmembrane potential $(V)$ is governed by the cable equation $\partial_{t} V(\mathbf{x}, t)=\nabla \cdot(D \nabla V)-\left[I_{\mathrm{fi}}(V, v)+\right.$ $\left.I_{\mathrm{so}}(V)+I_{\mathrm{si}}(V, w)\right] / C_{\mathrm{m}}$, where the ionic currents determine cell dynamics. The fast inward current $\left(I_{\mathrm{fi}}\right)$ represents the sum of the $\mathrm{Na}^{+}$ currents, the slow outward current $\left(I_{\text {so }}\right)$ represents the sum of the $\mathrm{K}^{+}$ currents, and the slow inward current $\left(I_{\mathrm{si}}\right)$ represents the sum of the $\mathrm{Ca}^{2+}$ currents. The membrane capacitance $\left(C_{\mathrm{m}}\right)$ is set to $1 \mu \mathrm{F} / \mathrm{cm}^{2}$. The diffusion tensor $(D)$ defines tissue structure and anisotropy (23). All simulations, except those in three dimensions, are isotropic, so that $D$ is a diagonal matrix whose off-diagonal elements are 0 and whose diagonal elements are $0.001 \mathrm{~cm}^{2} / \mathrm{ms}$. In three-dimensional simulations, propagation is set to be three times faster along than across cardiac fibers.

The three phenomenological currents are given by the following equations:

$$
\begin{gathered}
I_{\mathrm{fi}}(V, v)=-v p\left(V-V_{\mathrm{fi}}\right)(1-V) / \tau_{d} \\
I_{\mathrm{so}}(V)=V(1-r)\left(1-v k_{2}\right) / \tau_{0}+r / \tau_{r}
\end{gathered}
$$

and

$$
I_{\mathrm{si}}(V, w)=-w\left[1+\tanh \left(k_{1}\left(V-V_{c}^{\mathrm{si}}\right)\right] /\left(2 \tau_{\mathrm{si}}\right)\right.
$$

and the two gate variables of the model, $v$ and $w$, follow first-order equations in time:

$$
\begin{gathered}
\partial_{t} v(\mathbf{x}, t)=(1-p)(1-v) / \tau_{v}^{-}(V)-p v / \tau_{v}^{+} \\
\partial_{t} w(\mathbf{x}, t)=(1-p)(1-w) / \tau_{w}^{-}-p w / \tau_{w}^{+}
\end{gathered}
$$

where $\tau_{v}^{-}(V)=(1-q) \tau_{v 1}^{-}+q \tau_{v 2}^{-}$and $p$, $q$, and $r$ are defined by

$$
p=\left\{\begin{array}{ll}
0 & \text { if } V<V_{c} \\
1 & \text { if } V \geq V_{c}
\end{array} \quad q=\left\{\begin{array}{ll}
0 & \text { if } V<V_{v} \\
1 & \text { if } V \geq V_{v}
\end{array} \quad r= \begin{cases}0 & \text { if } V<V_{r} \\
1 & \text { if } V \geq V_{r}\end{cases}\right.\right.
$$

In the equations, $V$ varies between 0 and $\sim 1.4$ and is rescaled here as $V_{\mathrm{m}}=100 \mathrm{~V}-85$ when comparing other models or experiments. Initial conditions are $V=0, w=1$, and $v=1$. Although the step functions $p, q$, and $r$ are used for simplicity, we have verified that all results hold when continuous functions, such as tanh functions, are used.

We use three different sets of parameters to construct what, for ease of discussion, we refer to throughout this study as models 1,2, and 3 (see Table 1). Models 1 and 2 are used to demonstrate electrotonic effects in the absence of memory and are constructed to have the same APD restitution curve (for APD voltage thresholds of $-60 \mathrm{mV}$ and lower), as shown in Fig. $1 A$. However, the shape of the AP for $V$ greater than $-60 \mathrm{mV}$ is different in the two models, thereby producing different electrotonic effects when coupled in tissue. When simulating in spatially extended systems, we further subdivide model 2 into models $2 a, 2 b$, and $2 c$ to incorporate different shapes of $\mathrm{CV}$ restitution curves. This is done by changing $\tau_{v 1}^{-}$to 20 for model $2 a$ and to 150 for model $2 c$, while leaving $\tau_{v 1}^{-}$unchanged at 100 for model $2 b$. Note that changing $\tau_{v 1}^{-}$affects only the $\mathrm{CV}$ restitution shape in this model and does not alter the APD restitution or affect dynamics in isolated cells. The three different shapes of $\mathrm{CV}$ restitution for model 2 are shown in Fig. $2 A$.

Model 3 is used to demonstrate the effects of short-term memory of previous activations $(19,30)$ on the order of seconds related to the adaptation to new cycle lengths $(22,34,53)$. We increased the effect of memory beyond what has been shown previously for some parameter regimes of this model (64) by slightly altering the $\mathrm{K}^{+}$current in the manner of Fox et al. (26), who showed that the rapid delayedrectifier $\mathrm{K}^{+}$current $\left(I_{\mathrm{Kr}}\right)$ can play a role in increasing or decreasing memory effects. Therefore, we change $I_{\mathrm{so}}$ to the form $I_{\mathrm{so}} V=V(1-$ $r)\left(1-v k_{2}\right) / \tau_{0}+r V y / \tau_{r}$, where the extra gate variable $y$ is used that evolves in time according to

$$
\partial_{t} y(\mathbf{x}, t)=p(1-y) / \tau_{y}^{+}-(1-p)(y-0.1) / \tau_{y}^{-}
$$

where the rest value of $y$ is 0.1 . As with model 2, model 3 is subdivided into model $3 a$, with $\tau_{v 1}^{-}=20$, and model $3 b$, with $\tau_{v 1}^{-}$ unchanged, to produce two different $\mathrm{CV}$ restitution curves (see Fig. $7 A)$. The effects of long-term memory associated with remodeling (59), which has a time scale of days, are not considered.

Two different types of restitution curves are discussed: S1-S2 restitution and dynamic restitution. The S1-S2 restitution curve is obtained by pacing at a given fixed cycle length (S1) until steady state is reached and then introducing a subsequent stimulus (S2) after a certain DI and recording that DI and the resulting APD. By varying the DI before the introduction of $\mathrm{S} 2$, a full restitution curve is obtained. The S1-S2 restitution curve depends on the S1 cycle length, and the protocol can be used to determine a family of S1-dependent restitution curves, which may be related to each other in a unique relation (20). The dynamic restitution curve is obtained by pacing at a fixed cycle length until steady state is reached, at which time a single DI-APD pair is recorded. During alternans, the last two DI-APD pairs are recorded, so that both the long and short APs are included $(35,46$, 64). This process is repeated for decreasing cycle lengths until conduction block occurs. APD restitution curves for isolated cells are obtained in the same way as in Refs. 46 and 57, and the spatially extended APD and CV restitutions are obtained in strips and rings of simulated tissue following the method described in Ref. 23.

The 18 parameters used in the various models are given in Table 1. Further description of model parameters and their functions can be found in Refs. 23 and 25.

\section{RESULTS}

Even when the slope of the APD restitution curve is $>1$, alternans can be suppressed. Here, we present results showing how electrotonic and memory effects can prevent the induction of alternans and how CV restitution in each case can further affect the development or suppression of alternans.

Suppression of alternans by electrotonic effects. To illustrate how electrotonic currents can affect alternans development, we

\begin{tabular}{|c|c|c|c|c|c|c|c|c|c|c|c|c|c|c|c|c|c|c|}
\hline & $\tau_{v}^{+}$ & $\tau_{v}^{-}$ & $\tau_{y 2}^{-}$ & $\tau_{w}^{+}$ & $\tau_{w}^{-}$ & $\tau_{d}$ & $\tau_{0}$ & $\tau_{r}$ & $\tau_{\mathrm{si}}$ & $\tau_{y}^{+}$ & $\tau_{y}^{-}$ & $k_{1}$ & $k_{2}$ & $V_{c}^{\mathrm{si}}$ & $V_{c}$ & $V_{r}$ & $V_{v}$ & $V_{\mathrm{fi}}$ \\
\hline Model 1 & 10 & 350 & 80 & 562 & 48.5 & 0.15 & 1.5 & 12.5 & 10 & & & 15 & 0 & 0.2 & 0.25 & 0.16 & 0.001 & 0.15 \\
\hline Model 2 & 10 & 100 & 20 & 800 & 45 & 0.15 & 1.5 & 31 & 26.5 & & & 10 & 1 & 0.7 & 0.25 & 0.6 & 0.05 & 0.11 \\
\hline Model 3 & 3.33 & 45 & 300 & 600 & 40 & 0.075 & 8.3 & 38 & 127 & 1,000 & 230 & 60 & 0 & 0.7 & 0.25 & 0.25 & 0.05 & 0.25 \\
\hline
\end{tabular}
use models 1 and 2. As described above, these models have different AP morphologies, with model 2 having a longer plateau and faster late repolarization than model 1. However, both models exhibit the same APD restitution curves when measured at repolarization thresholds less than $-60 \mathrm{mV}$ (Fig.

Table 1. Parameter values used in the ionic model to produce simulations included in this study 

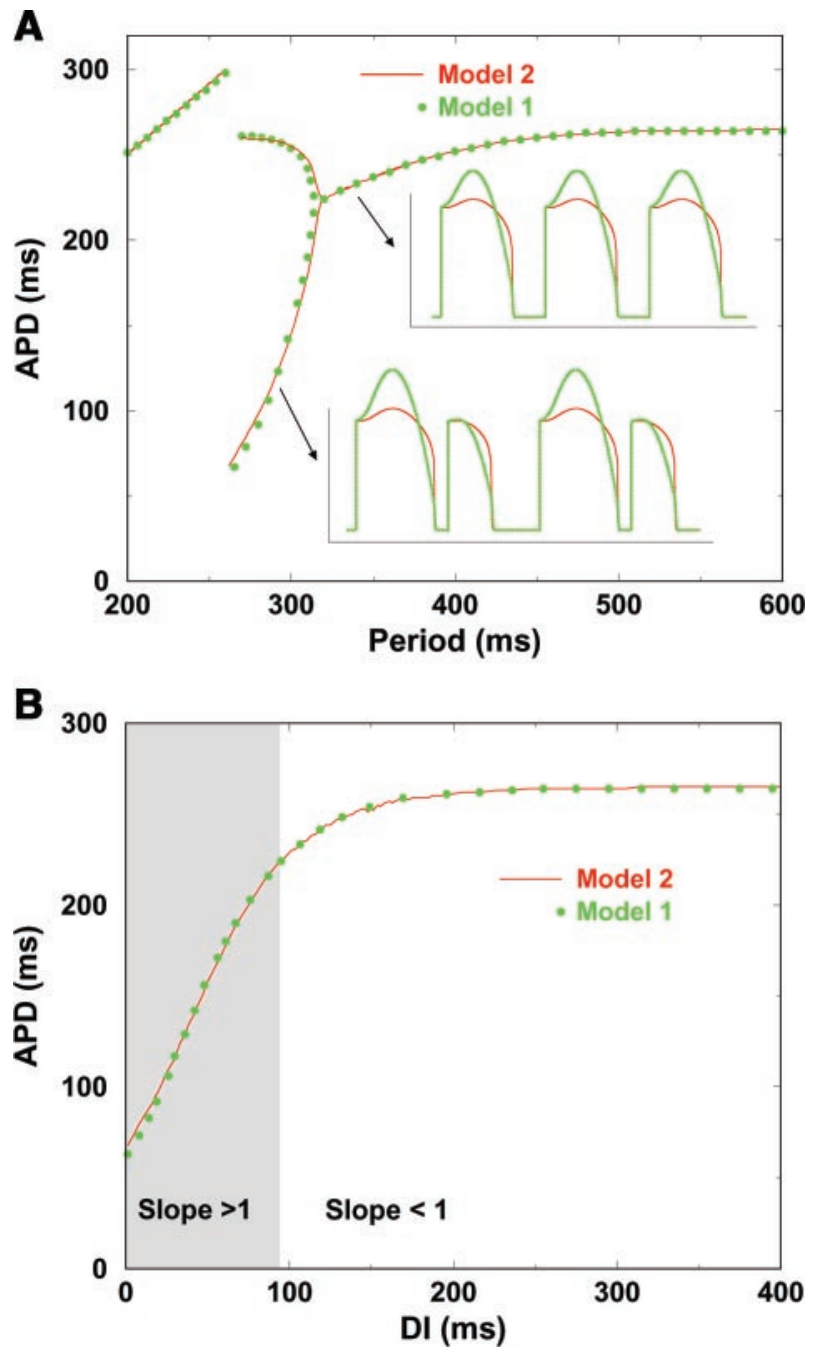

Fig. 1. A: action potential (AP) duration (APD) as a function of cycle length in an isolated cell for models 1 and 2. Alternans develops at shorter cycle lengths, where the slope of the APD restitution curve is $>1$. Insets, APs for models 1 and 2 during 1:1 and 2:2 rhythms, where model 1 is more triangular than model 2. B: APD restitution curves for models 1 and 2 in an isolated cell, with slope $>1$ [gray area; diastolic interval (DI) $<96 \mathrm{~ms}$ ]. Because models 1 and 2 do not include memory, dynamic and $\mathrm{S} 1-\mathrm{S} 2$ restitution curves for all $\mathrm{S} 1$ cycle lengths are identical.

1). In addition, both models are constructed so that they have no memory, and the APD restitution curve therefore is unique and independent of pacing protocol. Thus the APD depends only on the previous DI, and a map can be constructed as described by Guevara et al. (35), where alternans appears exactly when the slope of the restitution curve is $>1$ (51). In this case, because both models have the same APD restitution (Fig. 1B), alternans occurs in isolated cells for both models when the cycle length decreases below $318 \mathrm{~ms}(\mathrm{APD} \approx 222$ $\mathrm{ms}$, DI $\approx 96 \mathrm{~ms}$, slope $=1$ ), and the two models share the same DI range and voltage amplitudes of alternans and the same transition to $2: 1$ rhythm for cycle lengths $<260 \mathrm{~ms}$ (Fig. 1A).

The dynamics of alternans in tissue can vary from what is observed in isolated cells depending on CV restitution (18, 23, $29,67)$. Figure $2 A$ shows three different $C V$ restitution curves, corresponding to models $2 a, 2 b$, and $2 c$, which share the same
APD restitution but exhibit different dynamics when paced in a one-dimensional cable. Figure $2 B$ shows the spatial distribution of APDs obtained using the three models for two successive beats on a 10-cm-long cable that is paced from the left edge at a cycle length of $285 \mathrm{~ms}$. Although in an isolated cell
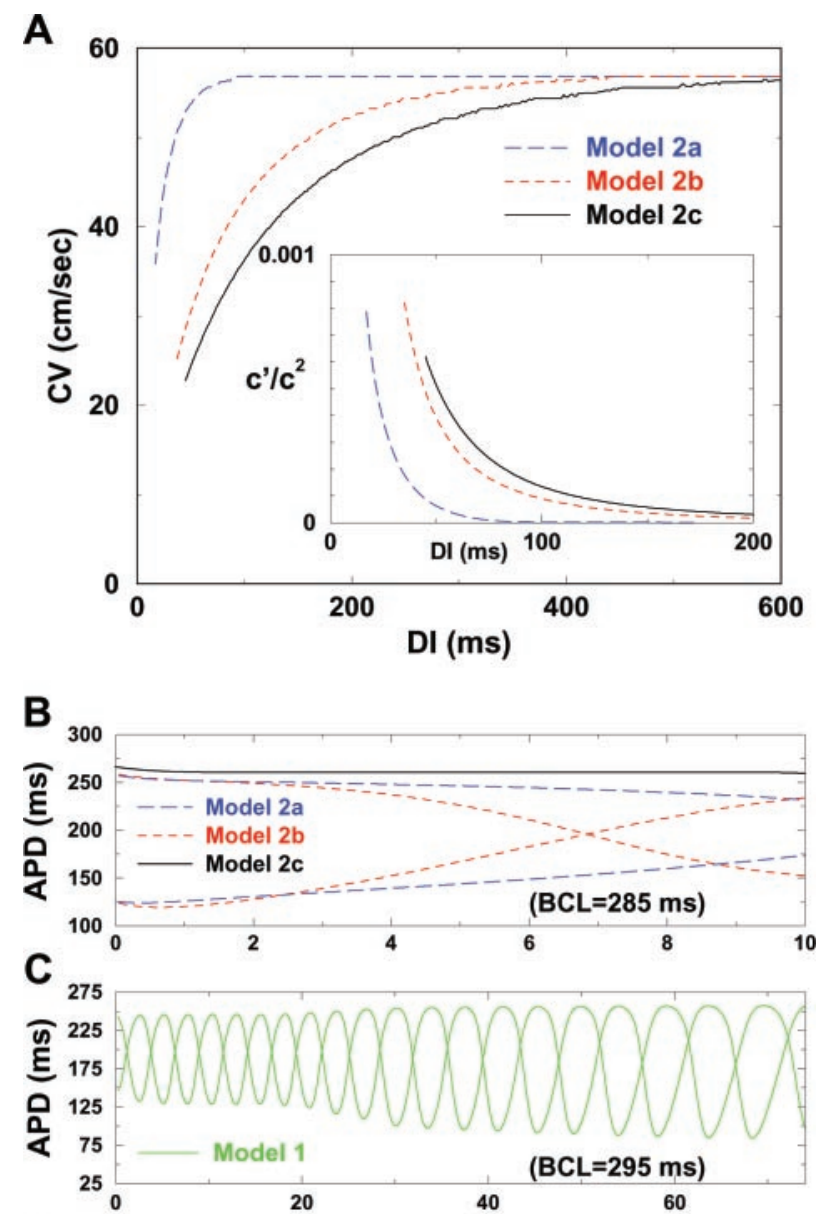

D

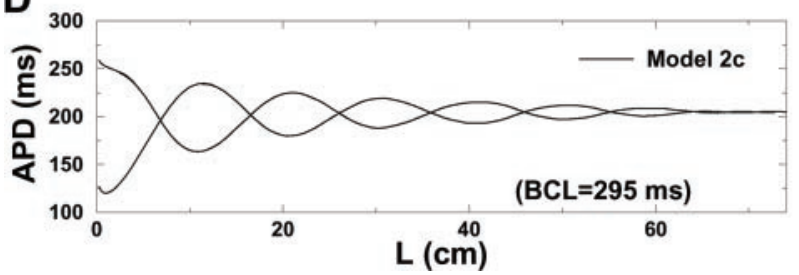

Fig. 2. A: conduction velocity $(\mathrm{CV})$ restitution curves corresponding to models $2 a, 2 b$, and $2 c$. Inset, ratio of the slope of the curve to the square of the CV, which is inversely proportional to the minimum length required to form discordant alternans (18). $B$ : alternans in a 10-cm-long 1-dimensional cable using the 3 different $\mathrm{CV}$ restitution curves shown in A. APD is shown as a function of length (L) on 2 successive beats after reaching steady state for a basic cycle length (BCL) of $285 \mathrm{~ms}$, with model $2 a$ producing concordant alternans, model $2 b$ discordant alternans with 1 node, and model $2 c$ conduction block leading to a $2: 1$ rhythm with a fixed APD of $270 \mathrm{~ms}$. $C$ : growth in alternans amplitude with distance from the pacing site for model 1 when paced at a cycle length of $295 \mathrm{~ms}$. Over time, this transient state evolves into a steady state with uniformly spaced nodes and equal amplitudes. $D$ : decrease in alternans. In contrast, when model $2 c$ is paced at the same cycle length of 295 $\mathrm{ms}$ in a cable of the same length, alternans decreases in amplitude as the distance from the pacing site increases. Note that steady state has been reached and alternans close to the pacing site persists at this period $(295 \mathrm{~ms})$ and does not develop conduction block, as for the shorter cycle length $(285 \mathrm{~ms})$ in $B$. In all cases, the pacing site is at the left boundary. 
the APD alternates between 107 and $257 \mathrm{~ms}$ for models $2 a, 2 b$, and $2 c$, in tissue the models exhibit concordant alternans, discordant alternans, and discordant alternans that progresses to conduction block (29) and 2:1 propagation, respectively.

Like CV restitution, electrotonic effects in tissue can alter the dynamics observed in isolated cells, because a cell with a membrane potential that is more negative (positive) than a neighboring cell will slightly repolarize (depolarize) that neighbor by diffusive currents. These effects can have important consequences during fast pacing and at boundaries. For example, models 1 and 2 have the same APD restitution, but model 2 repolarizes more quickly than model 1 and produces stronger electrotonic effects along the wave back. Therefore, even though models 1 and 2 exhibit the same pacing responses in isolated cells (Fig. 1A), differences can be observed in tissue. Although model 1 always exhibits alternans, regardless of changes in $\mathrm{CV}$ restitution, model 2 can suppress alternans. Figure $2 C$ shows discordant alternans for model 1 when paced at $295 \mathrm{~ms}$ on a cable, whereas Fig. $2 D$ shows alternans suppression far from the pacing site for model $2 c$ when paced at the same period.

Alternans can be reduced or suppressed completely at all points when no external pacing is present, such as during reentry on a ring of tissue, depending on a combination of the $\mathrm{CV}$ restitution curve and its slope $\left(c^{\prime} / c^{2}\right)$ and the electrotonic currents. Figure $3 A$ shows the maximum and minimum APDs obtained on a ring as a function of the period (where the various periods of the pulse are obtained by changing the ring size) for models $2 a, 2 b$, and $2 c$, which have different $\mathrm{CV}$ restitutions (Fig. 2A). For model $2 a$, alternans occurs over nearly the full range of periods for which alternans occurs in an isolated cell (small filled circles in Fig. 3A). The more sloping $\mathrm{CV}$ restitution of model $2 b$ also gives rise to alternans in a ring, but both the range of periods and the range of APDs over which it occurs are reduced. For model $2 c$, which has more pronounced CV restitution over a broader range of DIs (larger $c^{\prime} / c^{2}$ ), alternans is completely suppressed over the full range of periods in a ring because of increased electrotonic currents that stabilize the APD and prevent alternans induction. This effect of alternans suppression as a function of $c^{\prime} / c^{2}$ is described further in the DISCUSSION. Figure $3 A$, inset, also shows the spatial profile of the pulse along the ring at one instant in time for four different periods using model $2 c$ (first column, solid line) and model $2 a$ (second column, long dashes).

Figure $3 B$ shows the APD restitution curves obtained using model $2 c$ in an isolated cell, a one-dimensional cable, and a one-dimensional ring. Although there is no memory, several differences in the restitution curves arise because of coupling. First, the restitution curve obtained in an isolated cell can have a DI as small as 0 , because the cell can be excited at any time, provided a strong external stimulus is applied, whereas in one dimension there is a minimum DI below which propagation is not possible for any stimulus strength. Second, differences between the one-dimensional cable (long dashes) and ring (short dashes) appear at short DIs as a result of electrotonic effects, which are weaker in the cable near the pacing site. [The alternans amplitude at the pacing site in Fig. $2 D$ is similar to that of an isolated cell (Fig. 1A).] In the ring, the lack of external pacing allows $\mathrm{CV}$ restitution and electrotonic effects to eliminate alternans (17) [similar to the suppression of alternans far from the pacing site in a cable (Fig. 2D)], thereby
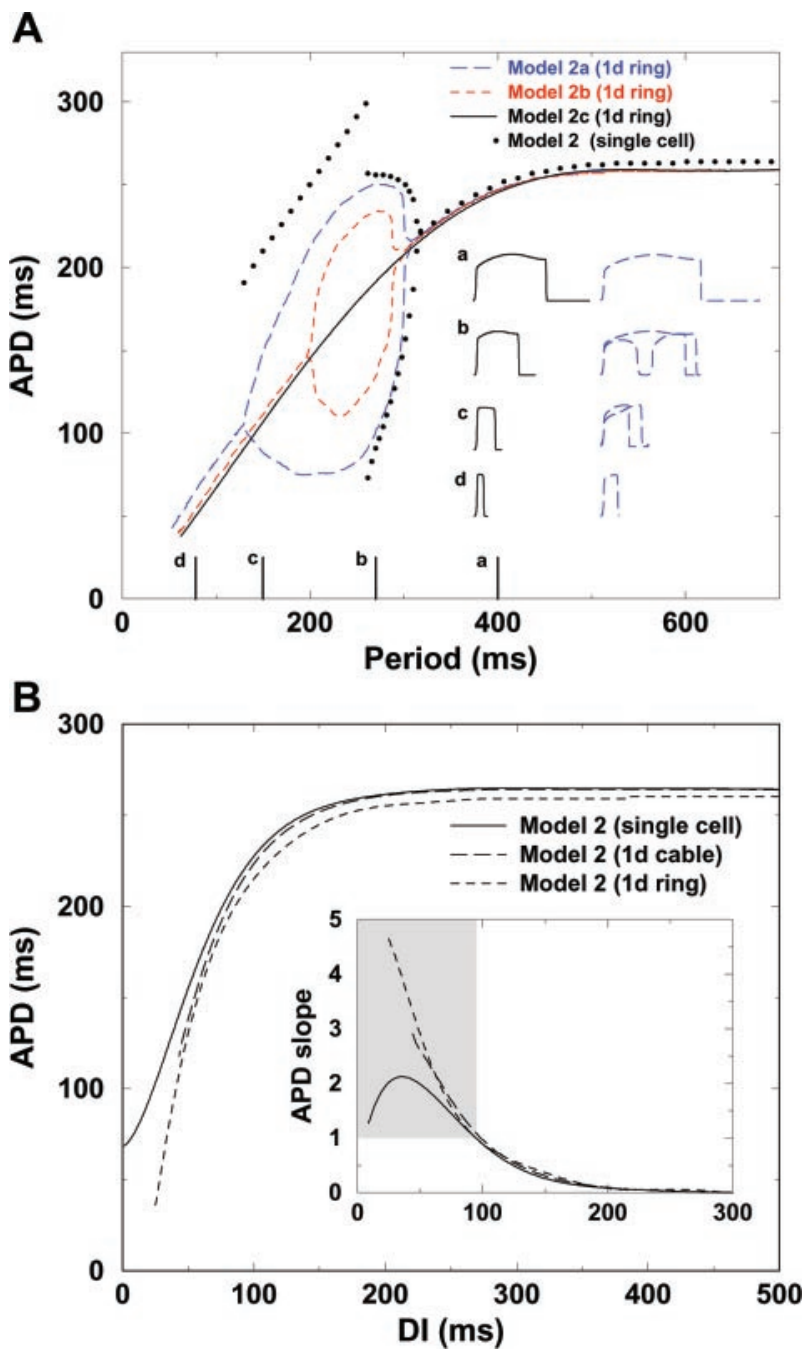

Fig. 3. A: APD alternans and its suppression as a function of period for a 1-dimensional ring using the $3 \mathrm{CV}$ restitution curves of Fig. $2 B$, where each period corresponds to a simulation using a different ring size. When the $\mathrm{CV}$ decreases abruptly at very short DIs (model $2 a$ ), alternans occurs over a range of cycle lengths, but when the CV decreases gradually over a wide range of DIs, the alternans can be decreased in amplitude and in DI range (model $2 b$ ) or even suppressed entirely (model $2 c$ ). Inset: spatial profiles along the ring using models $2 c$ and $2 a$ for 4 different ring sizes corresponding to periods of $400(a), 270(b), 150(c)$, and $76(d)$ ms. Note that although no alternans occurs for model $2 c$, alternans gives rise to different spatial profiles for model $2 a$ for periods of 270 and $150 \mathrm{~ms}$. B: APD restitution curves using model $2 c$ obtained for an isolated (single) cell, a 1-dimensional cable (1d cable), and a 1-dimensional ring (1d ring). Differences between the cable and ring restitutions become pronounced at short DIs because of electrotonic effects. Inset: slopes of the 3 restitution curves, with a maximum slope of 4.6 for the ring, which displayed no alternans at any period.

allowing adaptation to shorter cycle lengths without reaching alternans and conduction block compared with a cable. Therefore, a steeper restitution is obtained in the ring. It is important to note that although the maximum APD restitution slope obtained in the ring is 4.6, no alternans occurred for any period.

As in the one-dimensional case, the difference in AP shapes between model 1 and model 2 is enough to produce substantial differences in dynamics in two dimensions. A spiral wave initiated using model 1 , which has a shorter plateau and more gradual repolarization, develops discordant alternans and sustained breakup (Fig. 4A). Model 2a, however, with its longer 
A

Fig. 4. A: simulation of spiral wave dynamics using model 1. Discordant alternans develops away from the core as the wave rotates, as seen in frames 3 and 4 (middle), where the nodes separating long from short APs move as the wave rotates. Eventually, the alternans leads to conduction block and sustained breakup. Times are 1.8, 2.8, 9.5, $9.6,13.7$, and 14.3 s. $B$ : simulation of spiral wave dynamics using model 2 , which has a longer plateau and faster repolarization than model 1. Alternans is suppressed as a result of the increased coupling currents from the sharp wave back, and the induced spiral wave curls tightly and remains stable. Slight irregularities along the waves visible at the bottom are due to meandering of the tip trajectory and not to alternans. Times are 34, 74, 118, 188, 254, and $396 \mathrm{~ms}$. Excited tissue is shown in white and light gray and quiescent tissue in black. Tissue size is $12.5 \times 12.5 \mathrm{~cm}$ for $A$ and $17.5 \times 17.5 \mathrm{~cm}$ for $B$, with $\Delta x=0.025 \mathrm{~cm}$ and $\Delta t=0.05 \mathrm{~ms}$ for $A$ and $B$. Scale bars $=5 \mathrm{~cm}$. Movies of the still images shown in $A$ and $B$ are available in the online Data Supplement at the AJP-Heart web site.
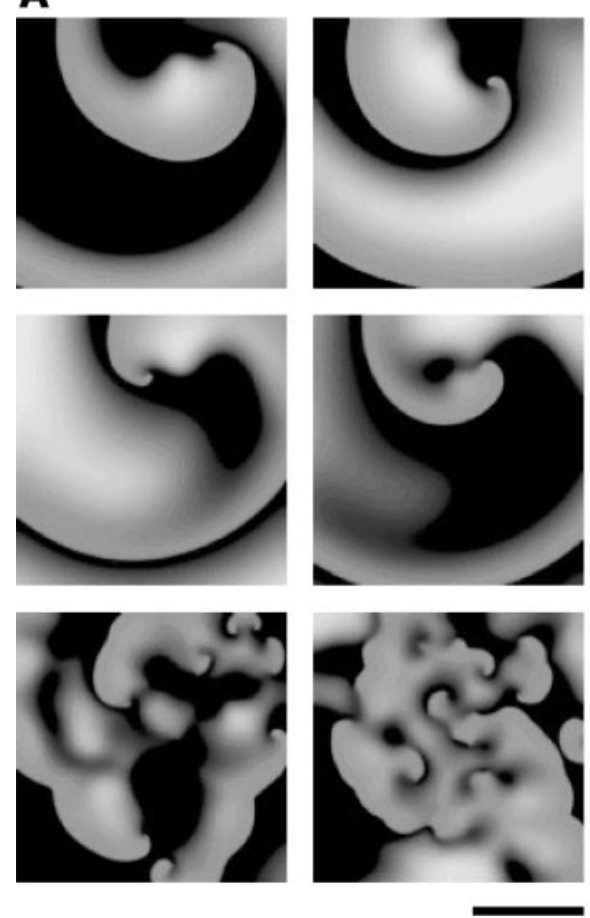

B
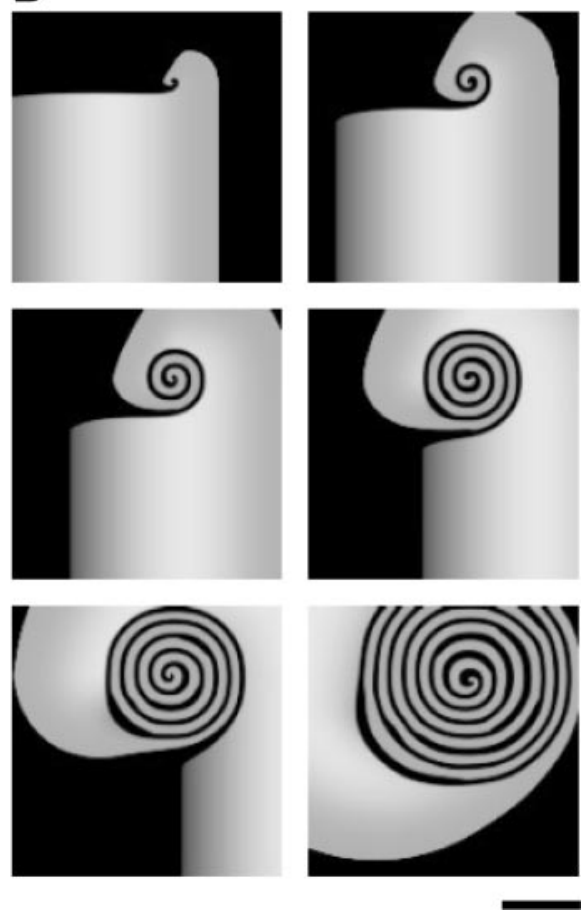

plateau and faster repolarization, is capable of winding into an extremely tight spiral with a period below $50 \mathrm{~ms}$ without developing any alternans. Note that as the spiral wave forms in Fig. $4 B$, it adjusts directly from a long AP to a series of short APs without alternans or conduction block. Similar stable behavior can be observed using models $2 b$ and $2 c$, because the spiral period is in the region with no alternans in the ring in all three cases. ${ }^{1}$

The same behavior also results when models 1 and 2 are simulated in a three-dimensional model of rabbit ventricles (65) that includes anisotropy and fiber directions. As in the two-dimensional case, model 1 gives rise to alternans, which initiates self-sustaining breakup throughout the ventricles (Fig. 5 , left). When models $2 a, 2 b$ (Fig. 5, right), and $2 c$ are used,

${ }^{1}$ The Data Supplement for this article (movies of Figs. 4, 5, and 8 as well as associated JAVA applets) is available online at http://ajpheart.physiology.org/ cgi/content/full/00747.2003/DC1. alternans does not occur, and an initiated spiral remains stable, despite steep APD restitution, even with the complex anatomy and fiber orientation of the ventricles.

Suppression of alternans by memory effects. Because it includes memory, model 3 , unlike models 1 and 2 , has a family of restitution curves resulting from different S1-S2 protocols as well as a distinct dynamic restitution curve. Figure 6 shows five different APD restitution curves obtained in an isolated cell using model 3, where the solid line represents the dynamic restitution curve and the four dashed lines represent S1-S2 curves obtained for $\mathrm{S} 1$ cycle lengths of $600,300,200$, and 100 ms. Although the slope of the dynamic APD restitution curve (Fig. 6, inset), which has been suggested as the most relevant to alternans onset $(35,46,57)$, is $>1$ for DI $<130 \mathrm{~ms}$ (maximum slope $=2.2$ ), no alternans occurs when the cells are paced at any cycle length. An improved criterion for determining the onset of alternans is the memory-corrected single-cell stability criterion of Tolkacheva et al. (64), which uses both the
Fig. 5. Electrotonic effects in rabbit ventricles (65) using models 1 (left) and $2 b$ (right). Spiral waves using model 1 break into multiple waves, whereas electrotonic effects arising from the faster-repolarizing AP of model 2 suppress alternans and prevent breakup of an initiated spiral wave, despite identical APD restitution curves with slope $>1$ for both models. Transmembrane potential is colored according to the color bar. Anisotropy ratio is $3: 1$, with $\Delta x=0.025 \mathrm{~cm}$ and $\Delta t=0.05 \mathrm{~ms}$. Scale bar $=1 \mathrm{~cm}$. Movies of the still images are available in the online Data Supplement at the AJP-Heart web site.
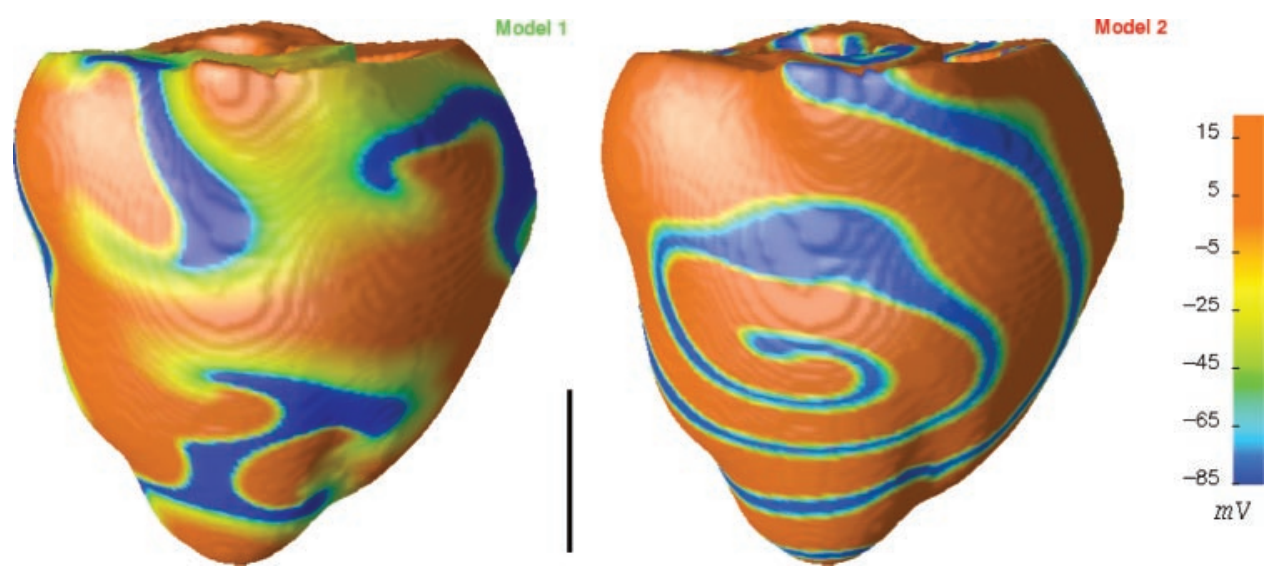


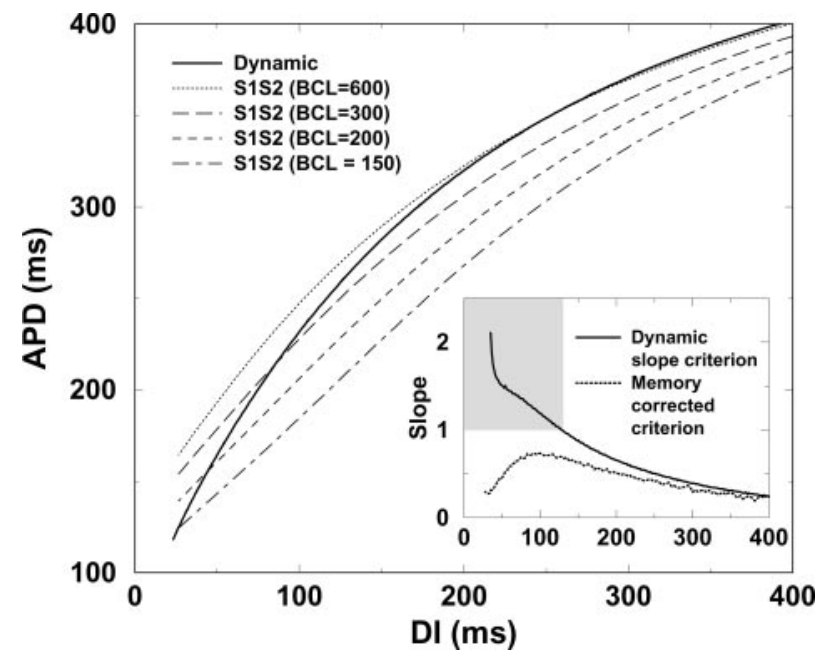

Fig. 6. Dynamic and S1-S2 restitution curves obtained using model 3. Significant memory effects cause the curves to differ and prevent the induction of alternans, despite dynamic restitution slope $>1$. Adding a memory correction to the APD restitution condition correctly predicts the absence of alternans for this case, despite dynamic restitution slope $>2$.

slope of the dynamic restitution curve and the slopes of the S1-S2 curves and predicts alternans when the criterion has a value $>1$. This criterion, shown over the same range of DIs in Fig. 6, inset, never exceeds 1 and, therefore, correctly predicts that alternans does not occur.

In tissue, changes to the $\mathrm{CV}$ restitution curve can influence whether alternans develops, even when no alternans is present in an isolated cell. As an example, Fig. 7A shows two different $\mathrm{CV}$ restitution curves used with model 3 , one flat over a large range of DIs but becoming steep at short DIs (model $3 a, \tau_{v 1}^{-}=$ 20 ) and the other more gently sloped over a larger range of DIs (model $3 b, \tau_{v 1}^{-}=45$ ). The dynamics obtained in a onedimensional cable when each model is paced at a cycle length of $154 \mathrm{~ms}$ is illustrated in Fig. 7B. Whereas model $3 a$ remains stable with no alternans, model $3 b$ exhibits alternans as a result of its more sloping CV restitution, which facilitates the development of alternans in long cables $(23,67)$. When the basic cycle length is decreased further to $153 \mathrm{~ms}$, model $3 b$ exhibits conduction block and 2:1 dynamics, whereas model $3 a$ remains stable (Fig. 7C). Furthermore, model $3 a$ does not produce alternans in a cable at any period of stimulation up to the minimum basic cycle length of $140 \mathrm{~ms}$ obtained at the minimum DI ( $\approx 23 \mathrm{~ms}$ ) of the model (Fig. 6).

In two dimensions, reentrant waves using either model $3 a$ or model $3 b$ do not produce alternans and are stable, even though the slope of the dynamic restitution curve is $>1$ (Fig. 8). Two snapshots during one rotation are shown using model $3 b$, where the spiral's period of rotation is about $170 \mathrm{~ms}$, which is well within the region of slope $>1$.

It is important to note that a transient breakup can still occur in some cases, even when alternans is suppressed. This breakup results from abrupt changes in cycle length, which can lead to conduction block as the cell tries to adjust to the new pacing but fails to capture the next beat. Therefore, when a reentrant wave is created using the memory model (particularly in model $3 a$ ), depending on initial conditions, conduction block can still occur in some cases after initiation as the reentrant wave turns, leading to breakup. Nevertheless, this breakup is not due to
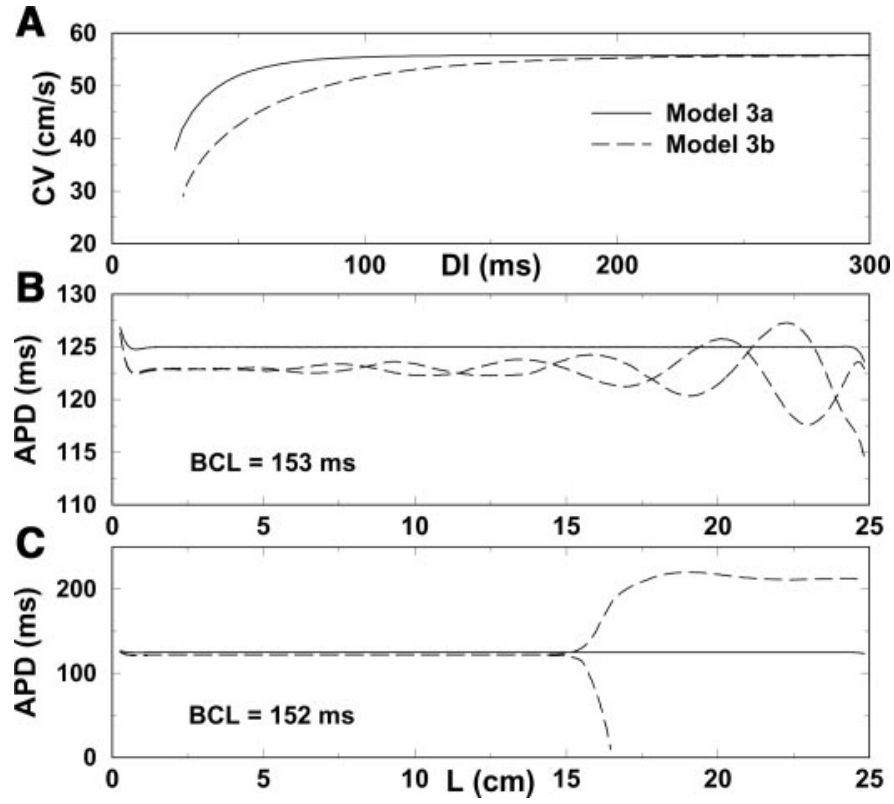

Fig. 7. A: CV restitution curves corresponding to models $3 a$ and $3 b . B$ : on a 1-dimensional cable paced from the left at a cycle length of $154 \mathrm{~ms}$, model $3 a$ remains stable, whereas model $3 b$ exhibits alternans whose amplitude increases with distance from the pacing site. APDs for 2 successive beats after reaching steady state are shown along the length of the cable. $C$ : when the cycle length is reduced to $153 \mathrm{~ms}$, model $3 a$ continues $1: 1$ conduction, whereas the alternans of model $3 b$ develops conduction block along the cable and transitions to 2:1 behavior far from the pacing site. Alternate beats 20 and 21 are shown. Over time, the site of the transition to 2:1 rhythm moves toward the pacing site at the left, resulting in 2:1 rhythm throughout the cable. Results were achieved by decreasing the BCL slowly to allow time for the cable to adjust to the new APD. In all cases, $\Delta x=0.025 \mathrm{~cm}$ and $\Delta t=0.1 \mathrm{~ms}$.

alternans, and, more importantly, it is not sustained and, therefore, does not lead to a continuous generation and annihilation of multiple reentrant waves as shown in Fig. $4 A$. Transient breakup of this nature generally does not occur when electrotonic effects in the absence of memory suppress alternans, inasmuch as the faster repolarization makes it easier for the wave front and back to adjust to sudden large changes in pacing cycle length without developing conduction blocks (17).
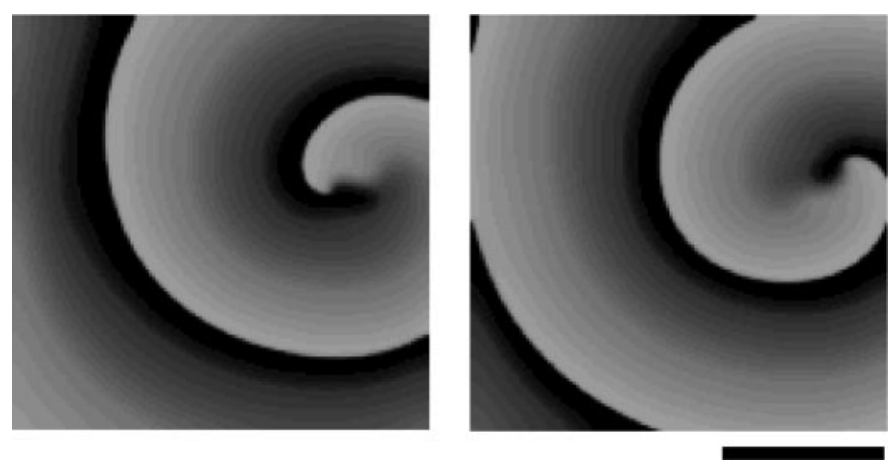

Fig. 8. Stable spiral wave obtained using model $3 b$, despite dynamic APD restitution curve slope $>1$. Two snapshots during 1 rotation are shown. Memory effects suppress alternans predicted by the APD restitution condition. Tissue size is $13 \times 13 \mathrm{~cm}$, with $\Delta x=0.025 \mathrm{~cm}$ and $\Delta t=0.1 \mathrm{~ms}$. Scale bar $=5 \mathrm{~cm}$. A movie of these still images is available in the online Data Supplement at the AJP-Heart web site. 


\section{DISCUSSION}

In this study, we show that diffusion currents due to the specific shape of the AP, memory of previous activations, and $\mathrm{CV}$ restitution can alter the criterion for the development of alternans when the slope of the restitution curve is $>1$. Variations of a phenomenological ionic cell model that separate electrotonic, memory, and $\mathrm{CV}$ restitution effects are used to illustrate these effects. However, the theory underlying the main points of the study, described below, can be interpreted independently of any particular model.

Alternans in isolated cells does not guarantee alternans in tissue. Even when alternans occurs in isolated cells, it is possible for electrotonic effects due to the AP shape, combined with $\mathrm{CV}$ restitution, to suppress alternans in tissue. Previous analytic studies using delay equations have shown that incorporating the role of $\mathrm{CV}$ restitution and electrotonic effects leads to a new criterion for the development of alternans (17, 18); specifically, alternans develops when the slope of the APD restitution curve exceeds unity by an amount that is a function of $\xi c^{\prime} / c^{2}$, where $\xi$ accounts for diffusion effects related to the AP morphology, especially its repolarization, and $c^{\prime} / c^{2}$ relates to the $\mathrm{CV}(c)$ and the slope of the $\mathrm{CV}$ restitution curve $\left(c^{\prime}\right.$; Fig. $2 A$, inset). Thus both large values of $\xi$, corresponding to strong electrotonic effects, and large values of $c^{\prime} / c^{2}$, corresponding to steeply sloped $\mathrm{CV}$ restitution or slow $\mathrm{CV}$, increase beyond 1 , the value that the slope of the APD restitution curve must achieve before alternans develops. Models 1 and 2, with different AP morphologies, have correspondingly different values of $\xi$ that change wave front-back interactions (17), causing differences in the formation of alternans in tissue, even though alternans is present in isolated cells in both cases. Therefore, model 1, which has small electrotonic effects along the wave back, exhibits discordant alternans throughout the cable (Fig. $2 C$ ), similar to previous numerical simulations (18, 23, 27, 28, 55, 67). However, model 2, with stronger electrotonic effects due to faster phase 3 repolarization, shows suppression of alternans far from the pacing site (Fig. 2D) when paced at the same cycle length, an effect that has not been demonstrated previously.

In contrast to the situation in a one-dimensional cable, where alternans is always present at the pacing site, in a one-dimensional ring where there is no pacing, alternans can be suppressed at all points during reentry. The faster the repolarization of the AP, the easier it is for alternans to be suppressed, as the value of $\xi$ becomes larger (17). However, CV restitution can diminish the stabilizing effect of $\xi$, which accounts for the different dynamics obtained using models $2 a, 2 b$, and $2 c$ in a ring (Fig. $3 A$ ). The ratio $c^{\prime} / c^{2}$ is relatively large over a wide range of DIs (Fig. 2A, inset) for model $2 c$; thus electrotonic currents completely suppress alternans in a one-dimensional ring (single-valued solid curve in Fig. 3A) for any ring size (period), because $\xi c^{\prime} / c^{2}$ remains large. On the other hand, when $c^{\prime} / c^{2}$ is small close to the onset of alternans in an isolated cell but increases at smaller DIs, alternans may be suppressed for sufficiently short periods (ring sizes), as for models $2 a$ and $2 b$, in which alternans is suppressed for periods below 130 and $196 \mathrm{~ms}$, respectively.

Similarly, electrotonic effects can be very important in determining the stability of reentrant waves in two and three dimensions, as shown in Figs. 4 and 5, where model 1 produces breakup and model 2 produces a stable spiral or scroll wave. [Breakup in model 1 no longer occurs when the tissue size is decreased below $7.5 \times 7.5 \mathrm{~cm}$, which indicates that alternans, rather than meander (3), is responsible for the breakup in this case, because discordant alternans can be prevented in smaller tissues $(18,23,67)$.$] Model 2$ can even produce an exceptionally tightly wound spiral, as shown for an extreme case in Fig. $4 B$, which while not typical of reentrant waves observed in cardiac tissue, has a frequency within values reported in the literature $(61,66)$. More realistic reentrant waves can be produced that similarly exhibit no alternans, despite a steep APD restitution by varying of some of the model parameters, such as the $\mathrm{Na}^{+}$conductance (23). Although, in three dimensions, thickness and fiber rotation $(25,56)$, as well as structure $(21,58)$, can destabilize reentrant waves with some specific dynamics, reentrant waves can remain stable when electrotonic effects are very strong, even when initiated in a realistic model of rabbit ventricular anatomy with fiber anisotropy (Fig. 5, right).

AP shape may be one explanation for the results of Hall et al. (36) and Banville and Gray (2), where APD restitution slope $>1$ was measured but no alternans was observed. The repolarization of the AP in both rabbit and frog ventricular myocardium in those experiments is relatively fast and is similar to model 2. Furthermore, differences in AP shape may be important in determining the stability of reentrant waves, as illustrated in two recent studies. Samie et al. (61) showed stable reentry in a simulation of left ventricular tissue using a variation of the Luo-Rudy I model (49) with a large $I_{\mathrm{K} 1}$ and unstable reentry in right ventricular tissue with small $I_{\mathrm{K} 1}$, which produced an AP with slower repolarization than in the left ventricular tissue. Likewise, Hondeghem et al. (39) found that triangular APs with slower repolarization were more likely to be proarrhythmic.

Suppression of alternans in isolated cells does not guarantee suppression of alternans in tissue. Cardiac tissue's memory of previous activations can prevent alternans in isolated cells, even when the APD restitution curve has slope $>1$. Analytic studies $(26,64)$ have shown how incorporating memory can change the stability of the 1:1 rhythm and prevent alternans. In particular, Tolkacheva et al. (64) generalized the original analysis of Guevara et al. (35), which assumed that APD was a function only of the preceding DI, for a case that included memory of the previous APD as well (as in Refs. 12 and 53). Because of this short-term memory, the stability criterion of the 1:1 rhythm was found to depend not only on the slope of the dynamic restitution curve, but also on the entire family of S1-S2 restitution curves obtained at different S1 cycle lengths. The criterion for $1: 1$ stability under these conditions becomes $\left|1-S_{\mathrm{S} 1-\mathrm{S} 2}\left[1+\left(1 / S_{\text {dyn }}\right)\right]\right|<1$ and not simply $\left|S_{\text {dyn }}\right|<1$ as the one-dimensional map without memory predicts, where $S_{\mathrm{dyn}}$ is the slope of the dynamic restitution curve for a given cycle length and $S_{\mathrm{S} 1-\mathrm{S} 2}$ is the slope of the S1-S2 restitution curve of the same cycle length that crosses the dynamic restitution at the given DI. Although it requires further testing in other models and different experimental conditions, in this case the memorycorrected stability criterion for model 3 (Fig. 6, inset) never exceeds 1 and correctly predicts that alternans will not occur in an isolated cell, although the slope of the dynamic APD restitution curve is $>1$. 
Nevertheless, even when memory suppresses alternans in an isolated cell, alternans still may develop in tissue as a result of electrotonic effects related to the CV restitution. We have found this to be the case for the ionic model of Fox et al. (26), which in an isolated cell with the maximum conductance of $I_{\mathrm{Kr}}$ increased by a factor of 2 does not alternate, despite APD restitution slope $>1$ (26), but does exhibit alternans in onedimensional cables when paced at high frequencies. In the same way, model $3 b$ exhibits alternans in a one-dimensional cable, but not in an isolated cell. As described above, a CV restitution curve that is sufficiently steep over a wide range of DIs (as in model $3 b$, compared with model $3 a$ ) promotes the induction of discordant alternans, because the value of $c^{\prime} / c^{2}$, which is inversely proportional to the size of tissue needed for discordant alternans to develop, is large $(18,23,67)$. Thus pacing at a certain cycle length may induce discordant alternans and conduction block in a cable of a given length for model 3b, but not for model $3 a$ (Fig. 7).

$C V$ restitution can promote or suppress alternans in tissue. On the basis of the results shown here, steepening the CV restitution over a broad range of DIs may appear to be a useful strategy to prevent the induction of alternans. However, it is important to consider that the combination of $\mathrm{CV}$ restitution and tissue size determines whether APD alternans in isotropic tissue will be concordant or discordant $(18,23,67)$. For tissue paced at a constant period, discordant alternans requires a minimum tissue size that is inversely proportional to $c^{\prime} / c^{2}(18)$, so that when $c^{\prime} / c^{2}$ is small (Fig. 2, $A$ and $B$, model $2 a$ ), only concordant alternans is formed. When $c^{\prime} / c^{2}$ is larger (Fig. 2, $A$ and $B$, model $2 b$ ), discordant alternans can develop, and conduction block can occur as $c^{\prime} / c^{2}$ grows (Fig. 2, $A$ and $B$, model $2 c$ ). Even when no alternans occurs in an isolated cell due to memory, as in model 3, CV restitution in tissue can still induce discordant alternans and conduction block far from the pacing site when $c^{\prime} / c^{2}$ is large, as shown for model $3 b$ in Fig. 7, where $c^{\prime} / c^{2}$ is larger for model $3 b$ than for model $3 a$. Therefore, a dilemma arises when the effects of altering $\mathrm{CV}$ restitution are considered. On the one hand, for a fixed tissue size, discordant alternans is more likely to appear as $c^{\prime} / c^{2}$ increases $(18,23$, 67); on the other hand, in the presence of strong electrotonic effects, alternans suppression is also more likely to occur with large $c^{\prime} / c^{2}$ (17) (Fig. 2, $A$ and $D$, and Fig. 3A, model 2c). Therefore, steeper $C V$ restitution curves may promote the alternans suppression effect of a strongly repolarizing wave back, but, at the same time, steep CV restitution may facilitate the progression of any alternans that does occur from concordant to discordant.

Other mechanisms may cause fibrillation and alternans. It is important to note that although alternans is a mechanism that can lead to conduction block and initiation of VF, it is not the only one, and other possible mechanisms may need to be considered as well, especially because the induction of complex electrical activity resembling fibrillation has been shown to occur using mathematical cell models without steep APD restitution or alternans. For example, the Luo-Rudy I model with calcium speedup of a factor of 2 has flat APD restitution but still produces complex VF-like dynamics (23). Certain types of trajectories of reentrant waves have been shown to lead to conduction blocks and continuous generation of multiple wavelets, despite flat APD restitution curves $(3,23)$. Tissue thickness $(6,23)$, rotational anisotropy $(23,56)$, and ventricular anatomy $(21,58)$ have also been shown to cause breakup for some types of reentrant wave trajectories, independent of APD restitution and cell model. Regional differences in ion channel density (61) as well as decreased conductivity and coupling $(7,44)$ have also been shown in some cases to lead to complex dynamics characteristic of VF. Even the results of experimental studies asserting that drugs that flatten APD restitution have antifibrillatory effects can be interpreted in other ways, so that other conditions may be involved in causing fibrillation. For instance, some of these studies $(31,52)$ also show an increase in the minimum DI, which itself has been suggested as an antifibrillatory target $(23,61)$ because a larger minimum DI can increase the core size and period of reentrant waves.

Similarly, steep APD restitution may not be the only mechanism that can produce alternans in cardiac tissue. A number of other possible mechanisms for alternans have been postulated, including tissue heterogeneities $(1,13,54)$; memory effects, even with APD restitution slope $<1(34,53)$; alternans in intracellular $\mathrm{Ca}^{2+}$ concentration $(13,14)$; and ischemia $(1,14$, 50 ), even though the APD restitution curve becomes flatter in ischemic tissue (63).

Limitations. Several limitations are associated with this study. Although for ease of discussion we have used a model that is able to separate electrotonic and memory effects, both are present in cardiac tissue and should be considered together. It was assumed here that memory did not extend to CV, although the maximum $\mathrm{CV}$ has been shown to become a function of cycle length during ischemia (38), and how CV memory would affect alternans development and suppression is unknown. Furthermore, we have not studied the effect of varying the minimum DI, which also may alter the conditions necessary for alternans to develop. Finally, the effects of tissue complexities, such as localized heterogeneities, transmural differences in ventricular cell types, and electromechanical coupling, were not considered.

Another more general limitation arising in theoretical or experimental studies involving restitution is how to measure APD restitution curves. First, different choices of thresholds for defining the APD and DI may produce APD restitution curves with different shapes, and it is not known how to determine which threshold is most relevant for predicting alternans. Furthermore, because of electrotonic effects due to the cores of VF wavelets, small double potentials $(19,23,45)$ and short APDs (4) can be recorded each time a wave tip passes by when dynamic APD restitution curves are measured during VF. These double potentials can be identified as true activations, although they are not (68), and can add points to the restitution curve with very small APD and DI. Including these spurious points in the restitution curve often produces a steeply sloped region at very short DIs and, therefore, can result in an erroneously higher value for the slope (40). For example, Koller et al. (46) showed that the restitution obtained by feeding the signal from VF into tissue does not produce many of the extremely small-valued APDs obtained during VF, even after the stimulus intensity and temperature are increased, most likely because of short APDs resulting from electrotonic double potentials by wave tips in VF that cannot be present during pacing. In addition, data points from experiments are often scattered widely during VF, probably because of double potential recordings. To avoid both the problem of data from 
wave tips and data scattering, Fenton et al. (23) suggested the use of a density plot, a method that works well for numerical simulations but remains to be verified experimentally, especially if strong memory is present.

Conclusions. In this study, we have shown that even when the slope of the APD restitution curve is $>1$ over a large range of pacing cycle lengths, the generation of electrical alternans and conduction blocks are a function of the AP shape, cardiac memory, and CV restitution. Although memory effects can prevent alternans in single cells, electrotonic effects due to AP shape and $\mathrm{CV}$ restitution can suppress alternans in tissue, even when alternans is present in single cells, but they can also induce alternans in tissue, even when no alternans is present in single cells. The results presented here and recently by others $(17,26,64)$ give a possible explanation for the absence of alternans in some experiments, even when the slope of the restitution was observed to be $>1(2,36)$, and also support the finding of Hondeghem et al. (39) that prolonging APD while making the AP more triangular tends to be proarrhythmic, whereas predominantly prolonging the plateau may not be. Because the fundamental mechanisms underlying VF remain largely unknown and because the onset of alternans and the dynamics of reentrant waves in cardiac tissue cannot always be well predicted by only the APD and CV restitution curves, our results support the idea $(2,32,40)$ that designing antiarrhythmic drugs targeted at altering only the slope of the APD restitution curve may not be appropriate at this time.

\section{ACKNOWLEDGMENTS}

We gratefully acknowledge the hospitality of the Aspen Center for Physics, where useful discussions with D. Gauthier and R. Gilmour helped this research. We also thank E. Cytrynbaum, B. Echebarria, and M. Guevara for fruitful discussions.

Part of this work has been previously published in abstract form (24).

\section{GRANTS}

This research was facilitated through an allocation of advanced computing resources by the National Computational Science Alliance, through the support of the National Science Foundation, and the computations were performed in part on the National Science Foundation Terascale Computing System at the Pittsburgh Supercomputing Center. We acknowledge the National Biomedical Computation Resource (National Institutes of Health Grant P41 RR-08605). This research was supported in part by National Science Foundation Grant PHY99-07949.

\section{REFERENCES}

1. Arce H, Xu A, Gonzalez H, and Guevara MR. Alternans and higherorder rhythms in an ionic model of a sheet of ischemic ventricular muscle. Chaos 10: 411-426, 2002.

2. Banville I and Gray RA. Effect of action potential duration and conduction velocity restitution and their spatial dispersion on alternans and the stability of arrhythmias. J Cardiovasc Electrophysiol 13: 1141-1149, 2002.

3. Bär M and Eiswirth M. Turbulence due to spiral breakup in a continuous excitable medium. Phys Rev E 48: R1635-R1637, 1993.

4. Beaumont J, Davidenko N, Davidenko JM, and Jalife J. Spiral waves in two-dimensional models of ventricular muscle: formation of a stationary core. Biophys $J$ 75: 1-14, 1998.

5. Beeler GW and Reuter H. Reconstruction of the action potential of ventricular myocardial fibres. J Physiol 268: 177-210, 1977.

6. Biktashev VN, Holden AV, and Zhang H. Tension of organizing filaments of scroll waves. Philos Trans R Soc Lond A Math Phys Sci 347: 611-630, 1994.

7. Bub F, Shrier A, and Glass L. Spiral wave generation in heterogeneous excitable media. Phys Rev Lett 88: 058101, 2002.
8. Cherry EM, Fenton F, Hastings HM, Xie F, Garfinkel A, Weiss JN, and Evans SJ. The role of decreased conduction velocity in the initiation and maintenance of atrial fibrillation in a computer model of human atria (Abstract). Pacing Clin Electrophysiol 24: II-538, 2002.

9. Cherry EM, Fenton FH, Hastings HM, and Evans SJ. Differences in reentry dynamics between two human atrial cell models (Abstract). Pacing Clin Electrophysiol 26: II-985, 2003.

10. Cherry EM, Rappel WJ, Evans SJ, and Fenton FH. Effects of wall heterogeneity in an anatomically realistic model of canine ventricles: a simulation study (Abstract). Pacing Clin Electrophysiol 26: II-1109, 2003.

12. Chialvo DR, Michaels DC, and Jalife J. Supernormal excitability as a mechanism of chaotic dynamics of activation in cardiac Purkinje fibers. Circ Res 66: 525-545, 1990.

13. Choi BR and Salama G. Simultaneous maps of optical action potentials and calcium transients in guinea-pig hearts: mechanisms underlying concordant alternans. J Physiol 529: 171-188, 2000.

14. Chudin E, Goldhaber J, Garfinkel A, Weiss J, and Kogan B. Intracellular $\mathrm{Ca}^{2+}$ dynamics and the stability of ventricular tachycardia. Biophys J 77: 2930-2941, 1999.

15. Courtemanche M. Complex spiral wave dynamics in a spatially distributed ionic model of cardiac electrical activity. Chaos 6: 579-600, 1996.

16. Courtemanche M, Ramirez RJ, and Nattel S. Ionic mechanisms underlying human atrial action potential properties: insights from a mathematical model. Am J Physiol Heart Circ Physiol 275: H301-H321, 1998.

17. Cytrynbaum E and Keener JP. Stability conditions for traveling pulse: modifying the restitution hypothesis. Chaos 12: 788-799, 2002.

18. Echebarria B and Karma A. Instability and spatiotemporal dynamics of alternans in paced cardiac tissue. Phys Rev Lett 88: 208101, 2002.

19. Efimov IR, Sidorov V, Cheng Y, and Wollenzier B. Evidence of three-dimensional scroll waves with ribbon-shaped filament as a mechanism of ventricular tachycardia in the isolated rabbit heart. J Cardiovasc Electrophysiol 10: 1452-1462, 1999.

20. Elharrar V and Surawicz B. Cycle length effect on restitution of action potential duration in dog cardiac fibers. Am J Physiol Heart Circ Physiol 244: H782-H792, 1983.

21. Fenton F, Cherry EM, Banville I, Gray RA, Hastings HM, Karma A, and Evans S. Validation of realistic 3D computer model of ventricular arrhythmias with optical mapping experiments (Abstract). Pacing Clin Electrophysiol 24: II-538, 2002.

22. Fenton F, Evans S, and Hastings H. Memory in an excitable medium: a mechanism for spiral wave breakup in the low excitable limit. Phys Rev Lett 83: 3964-3967, 1999.

23. Fenton FH, Cherry EM, Hastings HM, and Evans SJ. Multiple mechanisms of spiral wave breakup in a model of cardiac electrical activity. Chaos 12: 852-892, 2002.

24. Fenton FH, Cherry EM, Hastings HM, and Evans SJ. The APD restitution hypothesis revised: slope $>1$ does not always determine alternans and spiral wave breakup (Abstract). Pacing Clin Electrophysiol 26: II-1026, 2003.

25. Fenton FH and Karma A. Vortex dynamics in three-dimensional continuous myocardium with fiber rotation: filament instability and fibrillation. Chaos 8: 20-47, 1998.

26. Fox JJ, Bodenschatz E, and Gilmour RF Jr. Period-doubling instability and memory in cardiac tissue. Phys Rev Lett 89: 138101, 2002.

27. Fox JJ, Gilmour RF, and Bodenschatz E. Conduction block in onedimensional heart fibers. Phys Rev Lett 89: 198101, 2002.

28. Fox JJ, McHarg JL, and Gilmour RF Jr. Ionic mechanism of electrical alternans. Am J Physiol Heart Circ Physiol 282: H516-H530, 2002.

29. Fox JJ, Riccio ML, Hua F, Bodenschatz E, and Gilmour RF Jr. Spatiotemporal transition to conduction block in canine ventricle. Circ Res 90: 289-296, 2002.

30. Franz MR, Swerdlow CD, Liem LB, and Schaefer J. Cycle length dependence of human action potential duration in vivo. Effects of single extrastimuli, sudden sustained rate acceleration and deceleration, and different steady-state frequencies. J Clin Invest 82: 972-979, 1988.

31. Garfinkel A, Kim YH, Voroshilovsky O, Qu Z, Kil JR, Lee MH, Karagueuzian HS, Weiss JN, and Chen PS. Preventing ventricular fibrillation by flattening cardiac restitution. Proc Natl Acad Sci USA 97: 6061-6066, 2000.

32. Gilmour RF Jr. Electrical restitution and ventricular fibrillation: negotiating a slippery slope. J Cardiovasc Electrophysiol 13: 1150-1151, 2002.

33. Gilmour RF Jr. A novel approach to identifying antiarrhythmic drug targets. Drug Discov Today 8: 162-167, 2003. 
34. Gilmour RF Jr, Otani NF, and Watanabe M. Memory and complex dynamics in canine cardiac Purkinje fibers. Am J Physiol Heart Circ Physiol 272: H1826-H1832, 1997.

35. Guevara MR, Ward G, Shrier L, and Glass L. Electrical alternans and period doubling bifurcations. Comput Cardiol 11: 167-170, 1984.

36. Hall MG, Bahar S, and Gauthier DJ. The prevalence of rate-dependent dynamics in cardiac tissue. Phys Rev Lett 82: 2995-2998, 1999.

37. Hering HE. Experimentelle studien an saugethieren uber das electrocardiogram. Z Exp Pathol Ther 7: 363-378, 1909.

38. Hiramatsu Y, Buchanan JW Jr, Knisley SB, Koch GG, Kropp S, and Gettes L. Influence of rate-dependent cellular uncoupling on conduction change during simulated ischemia in guinea pig papillary muscles: effect of verapamil. Circ Res 65: 95-102, 1989.

39. Hondeghem LM, Dujardin K, and Clerck FD. Phase 2 prolongation in the absence of instability and triangulation, antagonizes class III proarrhythmia. Cardiovasc Res 50: 345-353, 2001.

40. Ideker RE, Rogers JM, and Gray RA. Steepness of the restitution curve: a slippery slope? J Cardiovasc Electrophysiol 13: 1173-1175, 2002.

41. Kalter HH and Schwartz ML. Electrical alternans. NY State J Med 48: 1164-1166, 1948.

42. Karma A. Electrical alternans and spiral wave breakup in cardiac tissue. Chaos 4: 461-472, 1994.

43. Karma A. New paradigm for drug therapies of cardiac fibrillation. Proc Natl Acad Sci USA 97: 5687-5689, 2000.

44. Keener JP. The effects of discrete gap junction coupling on propagation in myocardium. $J$ Theor Biol 148: 49-82, 1991 .

45. Kim YH, Garfinkel A, Ikeda T, Wu TJ, Athill CA, Weiss JN, Karagueuzian HS, and Chen PS. Spatiotemporal complexity of ventricular fibrillation revealed by tissue mass reduction in isolated swine right ventricle. J Clin Invest 100: 2486-2500, 1997.

46. Koller ML, Riccio MR, and Gilmour RF Jr. Dynamic restitution of action potential duration during electrical alterans and ventricular fibrillation. Am J Physiol Heart Circ Physiol 275: H1635-H1642, 1998.

47. Lewis T. Notes upon alternation of the heart. QJM 4: 141-144, 1911.

48. Lewis TJ and Guevara MR. Chaotic dynamics in an ionic model of the propagated cardiac action potential. J Theor Biol 146: 407-432, 1990.

49. Luo CH and Rudy Y. A model of the ventricular cardiac action potential: depolarization, repolarization, and their interaction. Circ Res 68: 1501$1526,1991$.

50. Murphy CF, Horner SM, Dick DJ, Coen B, and Lab MJ. Electrical alternans and the onset of rate-induced pulsus alternans during acute regional ischemia in the anaesthetised pig heart. Cardiovasc Res 32: 138-147, 1996

51. Nolasco JB and Dahlen RW. A graphic method for the study of alternation in cardiac action potentials. J Appl Physiol 25: 191-196, 1968

52. Omichi C, Zhou S, Lee MH, Naik A, Chang CM, Garfinkel A, Weiss JN, Lin SF, Karagueuzian HS, and Chen PS. Effects of amiodarone on wave front dynamics during ventricular fibrillation in isolated swine right ventricle. Am J Physiol Heart Circ Physiol 282: H1063-H1070, 2002.
53. Otani NF and Gilmour RF Jr. A memory model for the electrical properties of local cardiac systems. J Theor Biol 187: 409-436, 1997.

54. Pastore JM, Girouard SD, Laurita KR, Akar FG, and Rosenbaum DS. Mechanism linking T-wave alternans to the genesis of cardiac fibrillation. Circulation 99: 1385-1394, 1999.

55. Qu Z, Garfinkel A, Chen PS, and Weiss JN. Mechanisms of discordant alternans and induction of reentry in simulated cardiac tissue. Circulation 102: 1664-1670, 2000.

56. Rappel WJ. Filament instability and rotational tissue anisotropy: a numerical study using detailed cardiac models. Chaos 11: 71-80, 2001

57. Riccio ML, Koller ML, and Gilmour RF Jr. Electrical restitution and spatiotemporal organization during ventricular fibrillation. Circ Res 84: 955-936, 1999.

58. Rogers JM. Wave front fragmentation due to ventricular geometry in a model of the rabbit heart. Chaos 12: 779-787, 2002.

59. Rosen MR. What is cardiac memory? J Cardiovasc Electrophysiol 11: 1289-1293, 2000.

60. Rosenbaum DS, Jackson LE, Smith JM, Garan H, Ruskin JN, and Cohen RJ. Electrical alternans and vulnerability to ventricular arrhythmias. N Engl J Med 330: 235-241, 1994.

61. Samie FH, Berenfeld O, Anumonwo J, Mironov SF, Udassi S, Beaumont J, Taffet S, Pertsov A, and Jalife J. Rectification of the background potassium current, a determinant of rotor dynamics in ventricular fibrillation. Circ Res 89: 1216-1223, 2001.

62. Smith JM, Clancy EA, Valeri CR, Ruskin JN, and Cohen RJ. Electrical alternans and cardiac electrical instability. Circ Res 77: 110-121, 1988.

63. Taggart P, Sutton PM, Boyett MR, Lab M, and Swanton H. Human ventricular action potential duration during short and long cycles. Rapid modulation by ischemia. Circulation 94: 2526-2534, 1996.

64. Tolkacheva EG, Schaeffer DG, Gauthier DJ, and Krassowska W. Condition for alternans and stability of the $1: 1$ response pattern in a "memory" model of paced cardiac dynamics. Phys Rev E 67: 031904, 2003 .

65. Vetter FJ and McCulloch AD. Three-dimensional analysis of regional cardiac function: a model of rabbit ventricular anatomy. Prog Biophys Mol Biol 69: 157-183, 1998.

66. Warren M, Guha PK, Berenfeld O, Zaitsev A, Anumonwo JM, Dhamoon AS, Bagwe S, Taffet SM, and Jalife J. Blockade of the inward rectifying potassium current terminates ventricular fibrillation in the guinea pig heart. J Cardiovasc Electrophysiol 14: 621-631, 2003.

67. Watanabe MA, Fenton FH, Evans JE, Hastings HM, and Karma A. Mechanisms for discordant alternans. J Cardiovasc Electrophysiol 12: 196-206, 2001.

68. Witkowski FX, Plonsey R, Penkoske PA, and Kavanagh KM. Significance of inwardly directed transmembrane current in determination of local myocardial electrical activation during ventricular fibrillation. Circ Res 74: 507-524, 1994. 